\title{
EFFICIENT 1D AND CIRCULAR SYMMETRIC 2D FIR FILTERS WITH VARIABLE CUTOFF FREQUENCIES USING THE FARROW STRUCTURE AND MULTIPLIER-BLOCK
}

\author{
Carson K.S. Pun, S.C. Chan, and K. L. Ho \\ Department of Electrical and Electronic Engineering, \\ The University of Hong Kong, Pokfulam Road, Hong Kong.
}

\begin{abstract}
This paper proposes new structures for realizing ID and circular symmetric 2D FIR filters with variable cutoff frequencies. They are based on the interpolation of the impulse responses using the Farrow structure. The coefficients of the sub-filters in the Farrow structure are represented in sum-of-powers-of-two (SOPOT) coefficients, which can easily be implemented as simple shifts and additions. Furthermore, using the transposed form realization of the sub-filters, all the SOPOT coefficients can be implemented by a single multiplier-block exploiting the redundancy among the SOPOT coefficients. Several design examples are given to demonstrate the effectiveness and feasibility of the proposed approach.
\end{abstract}

\section{INTRODUCTION}

Farrow structure [1] is a very convenient method for implementing fractional delay digital filter (FD-DF), which is often required in applications like digital modem, time delay estimation. etc. It provides on-line tuning for the phase delay of the input signal. The basic idea is to approximate the impulse responses of FD-DFs with different delays using polynomial interpolation from impulse responses of a set of FD-DFs with fixed delays. To be more specific, a set of sub-filters with fixed delays $d_{i}, i=0 \ldots . L-1$ is first designed. Their impulse responses are then interpolated by a $L^{\text {th }}$ order polynomial using $d$ as the variable. So the resultant impulse response is the interpolated version of these impulse responses parameterized by the delay parameter $d$. Since the Farrow structure is based on interpolation of fixed set of impulse responses to provide on-line tuning of signal delay, it is natural to associate the parameter $d$ with other characteristics of a digital filter. In other words, the Farrow structure can also be used to realize digital filters with other tunable characteristics. For example, we can, not only realize a FD-DF with tunable delay, but also a FIR filter with tunable cutoff frequency using the Farrow structure. Again. a set of sub-filters with increasing cutoff frequencies in a given range is first designed. Then, their impulse responses are interpolated by polynomial approximation to obtain the impulse responses with different cutoff frequencies.

In this paper. we focus ourselves on the design of tunable linear-phase $1 D$ and 2D circular symmetric FIR filters with variable cutoff frequencies using the Farrow structure, though it is possible to generalize the technique to devise filters with other tunable characteristics. Moreover, a novel structure is proposed to reduce the implementation complexity of the sub-filters in the Farrow structure. More precisely, the filter coefficients of these sub-filters are represented in sum-of-powers-of-two (SOPOT) representation, which can be implemented with limited numbers of simple shift-add operations. Since the tunable filter might require slightly higher order of polynomial approximation, the number of sub-filters and the redundancies among their SOPOT coefficients will increase. To remove these redundant operations, the multiplier-block (MB) [3][4][5] is applied to the transposed form of the Farrow structure to further reduce the number of adders for its implementation. The design of the SOPOT subfilters is performed by means of a random search algorithm. which is able to determine very good candidates representing different tradeoff between arithmetic complexity and performance.

It should be noted that there are methods for designing FIR filters with variable cutoff frequencies. For a review of these techniques, please refer to pp. 356 of [2]. In brief, a prototype filter is first designed. A transformation is then used to determine the impulse response of the filter with the desired cutoff frequency. Some structure is able to perform on-line tuning of the cutoff frequency but the filters are limited to be linear-phase. The proposed method, on the other hand, is based on the polynomial interpolation of the impulse responses of a set of candidate filters. Therefore, it is applicable to both linear- and nonlinear-phase FIR filters. It also offers more flexibility in realizing tunable digital filters with other characteristics, at the expense of higher implementation complexity. Fortunately, using the SOPOT representation and the multiplier-block technique, the proposed structure is almost multiplier-free, except for the limited number of multipliers required for the final interpolation.

The rest of the paper is organized as follows: Section II is devoted to the theory, design and implementation of the tunable ID filter with variable cutoff frequency. Examples on tunable lowpass and bandpass filters with variable cutoff frequencies are given in Section III. The design of the circular symmetric 2D tunable lowpass filter using the McClellan transformation is also given. Finally, conclusions are drawn in Section IV.

\section{1-D FIR FILTERS WITH VARIABLE CUT- OFF FREQUENCIES}

The Farrow structure, fig 1, was originally proposed to realize variable fractional-delay digital filters with delay parameter $d$. More precisely, the output of the FD-DF, $y[(m+d) T]$, is given by

$$
y[(m+D+d) T]=\sum_{n=0}^{M} x[(m-n) T] \cdot h_{d}(n),
$$

where.$x[m T]$ is the input signal sampled at a period $T, h_{d}(n)$ is the FD-DF with delay $D+d$ and $D$ is an integer constant, and $M+1$ is the length of $h_{d}(n)$. To avoid the implementation of a large number of filters with different delays, Farrow [1] proposed to approximate each impulse response $h_{d}(n)$ with the following $L^{\text {th }}$ order polynomial in delay value $d$ such that the delay control is independent of the filter coefficients.

$$
h_{d}(n)=\sum_{k=0}^{L} c_{k \cdot n} d^{n} .
$$

The z-transform of (2) is then given by

$$
H_{d}(z)=\sum_{k=0}^{l} C_{k}(z) d d^{k}
$$

where $C_{k}(z)=\sum_{n=0}^{M} c_{k, n}=-1$ are the sub-filters. Thus the FD-DF with delay $d$ can be implemented by passing the signal through the sub-filters followed by the multiplication with the appropriate powers of $d$. As mentioned earlier, it is possible to associate the 
parameter $d$ with other characteristics of a digital filter, and implement this tunable filter using the Farrow structure.

To design a tunable lowpass filter with variable cut-off frequency using the Farrow structure, we first associate the increasing values of $d$ with a set of lowpass filters with increasing (or decreasing) cutoff frequencies in a given frequency range. Then, a set of lowpass FIR filters with cutoff frequency evenly sampled from $\omega_{c 1}$ to $\omega_{c_{2}}$ is designed say .using the Parks-McClellan algorithm, where $\omega_{c 1}$ and $\omega_{c 2}$ corresponding to the tunable range of the cutoff frequency. The next step is to interpolate each impulse response by a $L^{\text {th }}$ degree polynomial in $d$. To reduce the implementation complexity, the sub-filters are implemented as multiplier-less FIR filters using the SOPOT coefficients in the form

$$
\hat{c}_{k \cdot n}=\sum_{j=1}^{L_{s}} b_{k, n . j} \cdot 2^{a_{i}},
$$

with $b_{n, k, j}(i) \in\{-1.1\}$ and $a_{j} \in\{-l, \ldots,-1,0,1, \ldots . l\}$, where $l$ is a positive integer and its value determines the range of the coefficients, and $L_{s}$ is the number of terms used in the coefficient approximation and is usually limited to a small number. The coefficient multiplication can then be implemented as limited number of shifts and additions.

To design the SOPOT sub-filters, we minimize the $L_{\alpha}$ norm of its difference in frequency response with the idea one as shown in the following

$$
\delta_{a}=\max _{\omega \in S . d \in[0.1]}\left\langle\left|H_{i}\left(e^{j \omega}\right)-\hat{H}\left(e^{j \omega}\right)\right|\right\rangle .
$$

$H_{i}\left(e^{j \omega}\right)$ is the ideal frequency response and $\hat{H}\left(e^{j \omega \omega}\right)$ is the frequency response calculated for a given SOPOT filter coefficients. In other words, we try to minimize the peak ripple error $\delta_{a}$ for the whole frequency range of interest and the whole tunable range $d \in[0.1]$. The design procedure consists of two stages. First, the filter coefficients $b_{k, n . j}$ are optimized using a random search algorithm with respect to the criteria stated in (5). Then. the technique of multiplier-block is used to further reduce the number of adders required to implement all the SOPOT coefficients.

To be more specific, the real-valued coefficients $c_{k, n}$ are first determined by polynomial interpolation as mentioned previously in this section. Let $b$ be the vector containing these coefficients. Then the random search algorithm is to repetitively calculate a candidate SOPOT vector $b_{c}$ by adding to $b$ a random perturbation vector $\lambda b_{p}$ and then rounding it to the nearest SOPOT representation. That is,

$$
\boldsymbol{b}_{c}=\left\lfloor\boldsymbol{b}+\lambda \boldsymbol{b}_{p}\right\rfloor_{\text {SOPOT }} \text {. }
$$

The vector $b_{p}$ is a random vector with elements chosen in the range \pm 1 , and $\lambda$ is a user-defined variable used to control the size of the neighborhood to be searched. $[\cdot\rfloor_{\text {SOPOT }}$ is the rounding operator that converts every element inside the input vector to its closest SOPOT value with a given value of $l$. The performance measures $\delta_{a}$ of the new coefficients are then calculated. The set that yields the minimum peak error $\delta_{a}$ under the given constraints of total number of terms and $l$ is the optimum solution. Since this is a random search algorithm, the longer the searching time, the higher the chance of founding the optimal solution. There are several advantages of this algorithm. First of all, with the computational power of nowadays personal computer (PC) the time for obtaining high quality solutions is manageable. In fact, for the problem considered here, the overall design time only takes less than 10 minutes to complete on a typical Pentium-400 PC using Matlab 5.3, including both the design of SOPOT coefficients and the multiplier-block design. Secondly, it is applicable to problem with general objective function probably with very complicated inequality constraints. Moreover, a set of possible solutions representing different tradeoffs between computational complexity and performance will be generated during the search. We now consider the implementation of this tunable filter. In fig. 1 , it can be seen that a set of sub-filters $C_{k}(z), k=0,1,2 \ldots L$, has to be implemented, which is rather expensive. If each of these FIR filters is redrawn in transposed form (Fig. 2), then what we have to implement is the multiplication of the input sample with a large number of constant coefficients in SOPOT form. These products can efficiently be implemented using a technique called the multiplier-block. The basic idest of a multiplier block is to reduce the redundancies found in implementing all the SOPOT coefficients by removing any possible common sub-expressions in their representations. The generation of the multiplier-block in our case follows closely the work of [4].

\section{DESIGN RESULTS}

Example 1. Lowpass filter with tunable cutoff frequency In this example, a linear-phase lowpass filter with cutoff frequency tunable from $0.2 \pi$ to $0.4 \pi$ is designed. The transition bandwidth is $0.2 \pi$. The orders of the sub-filters and the interpolation polynomial are respectively 31 and 5 . Six subfilters with cutoff frequency equally spaced in the range from $0.2 \pi$ to $0.4 \pi$ are designed using the Parks-McClellan algorithm. After interpolation, the random search algorithm is used to determine the SOPOT coefficients of the sub-filters. The average number of terms in each SOPOT coefficients is found to be 3.05 , and the worst-case stopband attenuation is $43.03 \mathrm{~dB}$. Fig. 3 shows the frequency responses of the tunable filter at different control parameters. It can see that the frequency response is approximately equal-ripple in the stopband over the entire tuning range. Table 1 summarizes the design results of this tunable lowpass filter. Another point worth mentioning is that the multiplier-block (MB) is able to reduce the required number of adders to about one-third of its original value. Hence, the system complexity is dramatically reduced with only slight or negligible clegradation in performance. We have also designed a number of tunable filters using different orders of interpolation with the same filter specifications. Fig 4 plots the worst-case stopband attenuation as a function of the order of the interpolation polynomial.

\begin{tabular}{|l|c|}
\hline Interpolation order & 5 \\
\hline Filter order & 31 \\
\hline Cutoff frequency tuning range & $0.2 \pi-0.4 \pi$ \\
\hline Transition bandwidth & $0.2 \pi$ \\
\hline Worst-case stopband attenuation & $43.03 \mathrm{~dB}$ \\
\hline Average no. of terms of SOPOT coefficient & 3.05 \\
\hline Required adders before using MB & 241 \\
\hline Required adders after using MB & 77 \\
\hline
\end{tabular}

Table 1. Tunable lowpass filter details in Example 1.

\section{Example 2. Bandpass filter with tunable cutoff frequency}

In this example, we are going to design a bandpass filter with tunable cutoff frequency using the previous result on tunable 
lowpass filter. The basic idea is to cascade a tunable lowpass and a tunable highpass filters together so that we can control its cutoff frequencies individually. The proposed structure is illustrated in fig. 5. It can be seen that the filter bandwidth can be controlled by two variables: $d_{l p}$ and $d_{h p}$, which control the cutoff frequencies of the lowpass filter $h_{l p}[n]$ and the highpass filter $h_{h p}[n]$, respectively. It is possible to reduce the overall system delay by interpolating the filters in 2-dimension, but the overall complexity will increase dramatically with the square of the order used instead of linearly with the proposed cascade structure. Fig. 6 shows the frequency responses of the proposed tunable bandpass filter with different values of control parameters $d_{l p}$ and $d_{l p}$. The order of the sub-filters in the tunable lowpass and the tunable highpass filters are both 16 , while the order of the interpolation polynomial is 4 . The transition bandwidth and the tunable ranges of the first and second cutoff frequencies are $0.1 \pi,[0.6 \pi, 0.7 \pi]$, and $[0.1 \pi, 0.2 \pi]$, respectively. The worst-case stopband attenuation is around $30 \mathrm{~dB}$. The number of adders after the use of multiplier-block is 51 , which is about $36 \%$ of the original SOPOT sub-filters.

Example 3. Tunable 2-D circular symmetric filter using McClellan Transformation [6]

In this example, a circular symmetric zero-phase 2D FIR filter with variable cutoff frequency is designed. It is obtained by applying the following McClellan transformation [7]

$$
\begin{aligned}
& G\left(z_{1}, z_{2}\right)=-0.3955+0.25 \cdot\left(z_{1}+z_{1}^{-1}\right)+0.25 \cdot\left(z_{2}+z_{2}^{-1}\right), \\
& +0.098875 \cdot\left(z_{1}+z_{1}^{-1}\right) \cdot\left(z_{2}+z_{2}^{-1}\right)
\end{aligned}
$$

to the Farrow-based tunable 1-D zero-phase filter obtained in previous section. The structure of the resulting $2 \mathrm{D}$ filter is shown in fig. 7. Again. the coefficients of the sub-filters are represented in SOPOT form and are implemented as a multiplierblock. The 2D filter has a cutoff frequency $\omega_{s}=\sqrt{\omega_{1}^{2}+\omega_{2}^{2}}$ tunable from $0.2 \pi$ to $0.4 \pi$. The transition bandwidth is $0.2 \pi$. The order of the sub-filters is 16 while that of the interpolation polynomial is 4 . The frequency response of the $1 D$ prototype filter is shown in fig. 8, and the worst-case stopband attenuation is $30.51 \mathrm{~dB}$. The real-valued sub-filters require 85 multipliers, while the multiplierless sub-filters, without the use of the multiplier-block, require 182 adders. With the transposed form and the multiplier-block, the number of adders required can be dramatically reduced to 36 . Frequency responses of the tunable 2D circular symmetric FIR filter with different control parameters are shown in Fig 9.

\section{CONCLUSION}

New structures for realizing ID and circular symmetric 2D FIR filters with variable cutoff frequencies are presented. They are based on the interpolation of the impulse responses using the Farrow structure. The coefficients of the sub-filters in the Farrow structure are represented in sum-of-powers-of-two (SOPOT) coefficients. which can easily be implemented as simple shifts and additions. Furthermore, using the transposed form realization of the sub-filters, all the SOPOT coefficients can be implemented by a single multiplier-block exploiting the redundancy among the SOPOT coefficients. Several design examples are given to demonstrate the effectiveness and feasibility of the proposed approach.

\section{REFERENCES}

[1] C. W. Farrow. "A continuously variable digital delay element". Proceedings of ISCAS 88. pp. $2641-2645$.
[2] Sanjit K. Mitra. James F. Kaiser, "Handbook for Digital Signal Processing". Wiley, 1993.

[3] Potkonjak. M.: Srivastava, M.B.: Chandrakasan. A.P.. “" Multiple constant multiplications: efficient and versatile framework and algorithms for exploring common subexpression elimination". IEEE Trans. Computer-Aided Design of Integrated Circuits and Systems, vol. 15, pp. 151 -165. Feb. 1996.

[4] Andrew G. Dempster: Malcolm D. Macleod, "Use of minimunadder multiplier blocks in FIR Digital Filters", IEEE Trans. Circuits and Systems II: Analog and Digital Signal Processings. vol. 42. pp. $569-577$. Sept. 1995.

[5] Pasko. R.; Schaumont, P.; Derudder. V.: Vernalde, S.; Durackova. D., "A new algorithm for elimination of common subexpressions". IEEE Trans. Computer-Aided Design of Integrated Circuits and Systems. vol. 18. pp. 58 -68. Jan. 1999.

[6] James $\mathrm{H}$. McClellan. "The design of two-dimensional digital filters by transformations", Proc. $7^{\text {th }}$ Annual Princeton Conference, Information Sciences and Systems 1973, pp. 247-51.

[7] Psarakis. E.Z.: Moustakides. G.V. "Design of two-dimensional zero-phase FIR filters via the generalized McClellan transform". Trans. Circuits and Systems, vol..38, pp. 1355-1363. Nov. 1991. $X(-)$

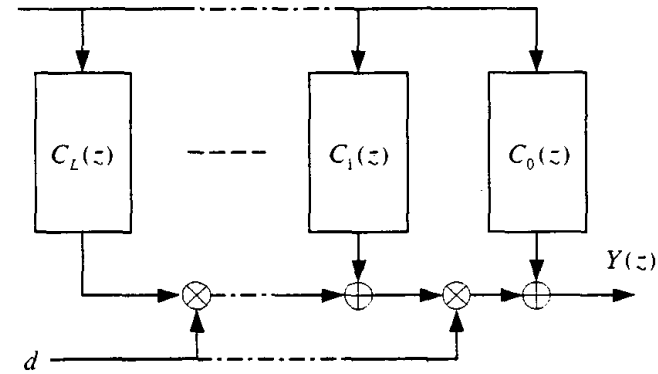

Figure 1. Original Farrow structure.

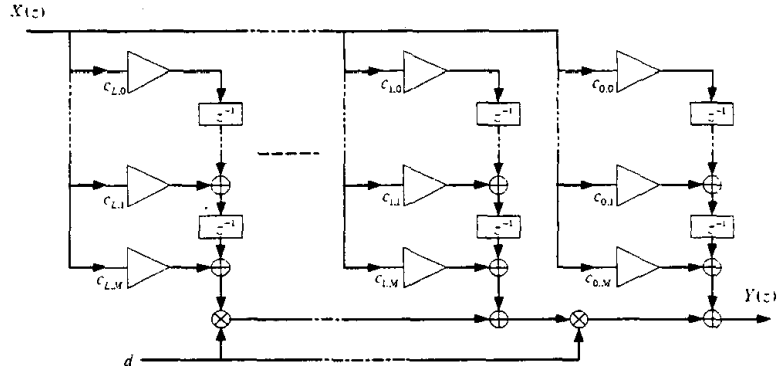

Figure 2. Proposed Farrow structure.

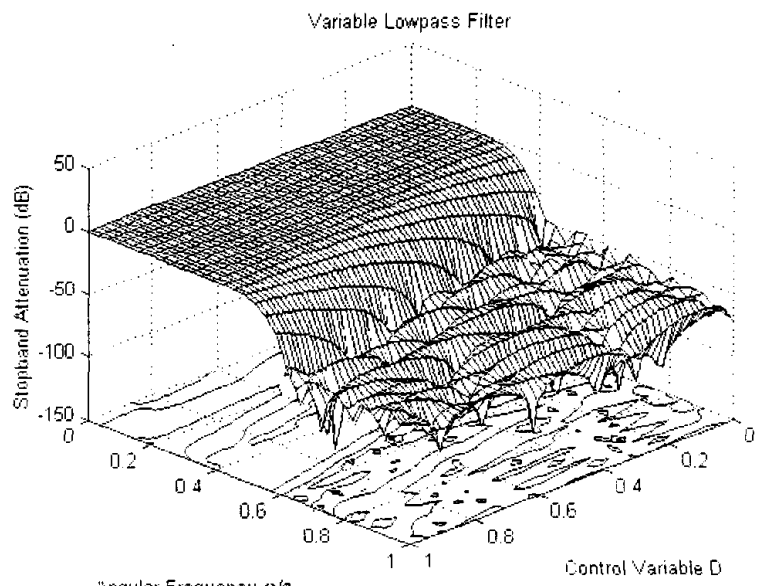

Figure 3. Frequency responses of the tunable cutoff frequency lowpass filter in Example 1. 


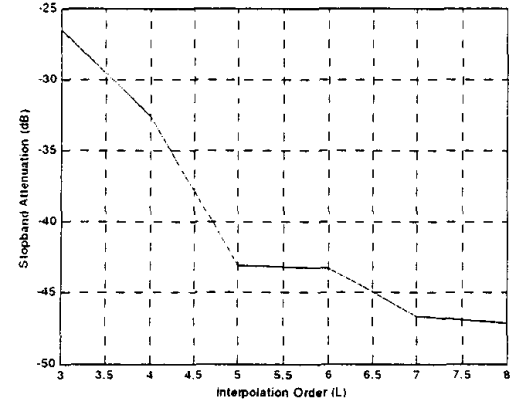

Figure 4. Worst-case stopband attenuation of the tunable cutoff frequency lowpass filter versus the order of the polynomial interpolation. (Example 1).

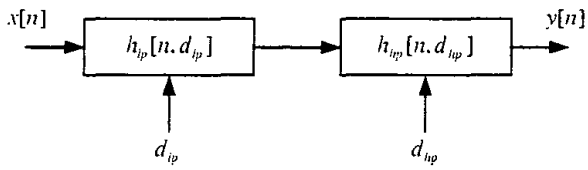

Figure 5. Tunable bandpass filter implemented as the cascade of two tunable lowpass and highpass filters (Example 2).

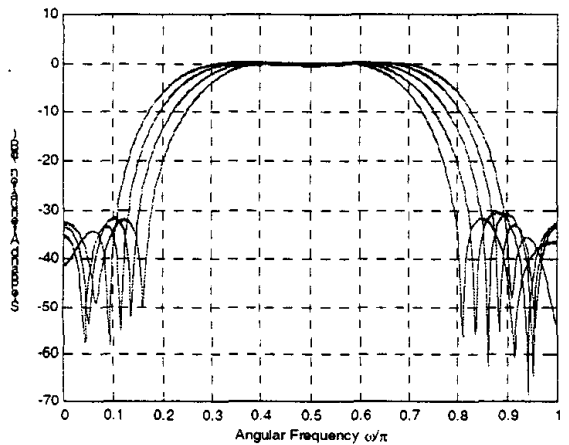

Figure 6. Frequency responses of the tunable bandpass filter with different control variables. (Example 2).

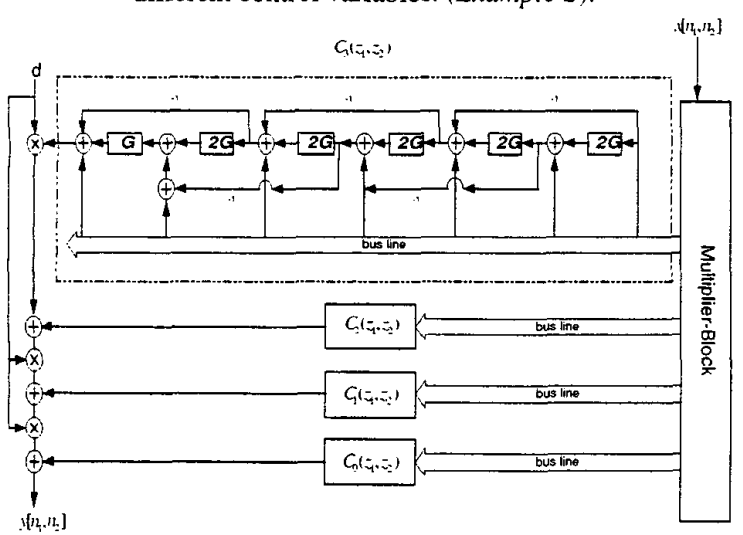

Figure 7. Proposed Farrow structure for 2D tunable cutoff frequency circular symmetric zero-phase filter. (Example 3).

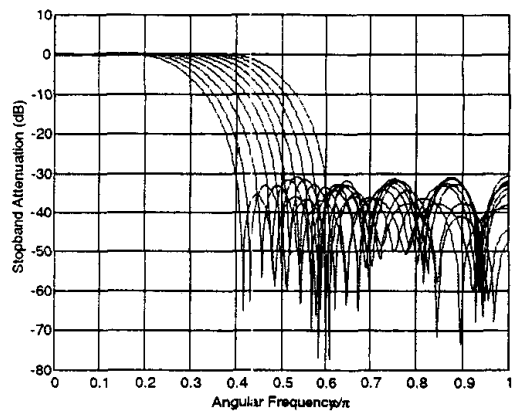

Figure 8. Frequency responses of the tunable ID prototype filter at different control variables. (Example 3).
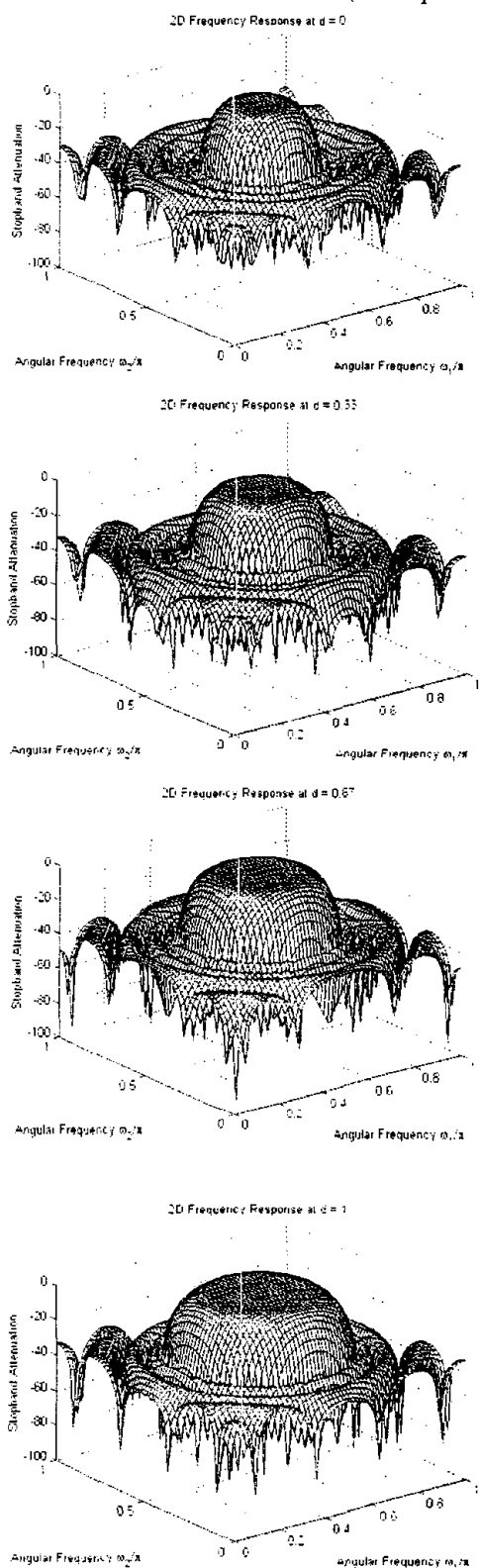

Figure 9. Frequency responses of the proposed tunable 2D filter at different control variables. $(d=0,0.33,0.67,1)$. 